\title{
Estimation of Heterosis for Yield and Quality traits in Bitter Gourd (Momordica charantia L.)
}

\author{
Amrita Kumari ${ }^{1}$, Sangeeta Shree ${ }^{1 *}$, Randhir Kumar ${ }^{1}$, Chandan Kishore ${ }^{2}$, \\ V. K. Singh ${ }^{1}$ and M. Haque ${ }^{3}$
}
${ }^{1}$ Department of Horticulture (Vegetables and Floriculture), Bihar Agricultural University, Sabour, 813210, India
${ }^{2}$ Department of Plant Breeding and Genetic, Bihar Agricultural University, Sabour, 813210, India
${ }^{3}$ Department of Agronomy Bihar Agricultural University, Sabour, 813210, India

*Corresponding author

\section{Ke ywords \\ Bitter gourd and Heterosis \\ Article Info \\ Accepted: \\ 08 January 2020 \\ Available Online: \\ 10 February 2020}

\section{A B S T R A C T}

\section{Introduction}

Bitter gourd (Momordica charantia L.) is a tender, edible fruit in the genus of Momordica which is a climbing vine belongs to the family cucurbitaceae. The genus Momordica has about 80 species among which Momordica charantia L. is most cultivated species having chromosome number $2 \mathrm{n}=22$. Eastern India or southern China consider as the center of origin of bitter gourd (Walters and DeckerWalters 1988; Miniraj et al., 1993). Bitter gourd is also known as 'karela' in Hindi which may be Dravion origin (Turner, 1966). Due to its pharmaceutical, antidiabetic property and high nutritive value in terms of ascorbic acid and iron it is considered as prized vegetable among cucurbits (Behera 2004).

Bitter gourd is highly cross pollinated crop and bears staminate and pistilate flowers separately on the same plant, i.e. monoecious which offers considerable amount of variability. In spite of the economic and medicinal importance of the crop, due 
attention was not paid towards a need based crop improvement programmed. Now a day, gradually the cultivation of bitter gourd has become popular, because of the growing awareness of the health among consumer. Varieties and hybrids of bitter gourd have been developed by the effort of many breeders but there is also need for its improvement to develop varieties or hybrids suited for earliness, high yield, improved postharvest quality, uniformity, and wider adaptability and resistant to disease and pest. Heterosis is the superiority of F1 Hybrid over the mean of the parents or over the better parent (BP) or over the standard check (Hayes et al., 1956) regarding traits.

The main aim of heterosis breeding is to achieve higher yield with better quality. The commercial exploitation of hybrid vigour depends on the ease with which the technique employed and cost of seed production. Bitter gourd being monoecious can be profitably utilized for the production of F1 hybrid seeds at cheaper price. High number of hybrid seeds per cross makes it more economical. Further, the crop being cultivated at wider spacing, the seed requirement per hectare for commercial cultivation would be low and cost effective. bitter gourd offers greater scope for exploitation of hybrid vigour on commercial scale to increase the productivity and production. The heterosis expressed types of gene action which helps in selection of breeding methodology for crop improvement. There are several scientists who worked on heterosis and obtained varying degree of existence of heterosis. Pal and Singh (1946); Lawende and Patil, 1989, Laxman et al., 2012 and Rani et al., 2014) and Thangamani and Pugalendhi (2012). The present experiment was undertaken to identify potential parental combinations that are likely to produce superior hybrids having qualities with maximum yield.

\section{Materials and Methods}

The present investigation was conducted at the research farm, Department of Horticulture (Vegetable and Floriculture) of Bihar Agricultural College, Sabour, Bhagalpur (Bihar) during two summer season of 2016 and 2017. The experimental material for this experiment comprised of seven promising and diverse genotypes i.e., PusaAusadhi, Jhalri, Kathi No.1, PusaRasdar, BRBT Local, Konkan Tara, Solan Hara NBGH 167 (Standard check). They were crossed in all possible combination in diallel fashion excluding reciprocals to get 21 hybrids. All the 29 treatments ( 7 parents, $21 \mathrm{~F}_{1}$ hybrids with one check) were grown in randomized block design with three replications with spacing of $2.0 \mathrm{~m} \times 0.5 \mathrm{~m}$ in plot size $4.0 \mathrm{~m} \mathrm{X}$ $3 \mathrm{~m}$. The recommended agronomic practices were adopted for raising the better crops. Observations were recorded on 20 economically important traits viz; node number at which first male flower appear, node number at which first female flower appear, Days taken to first male flower appear, Days taken to first female flower appear, days to $50 \%$ flowering, internodal length $(\mathrm{cm})$, number of primary branches per plant, vine length $(\mathrm{cm})$, number of fruits per plant, fruit weight $(\mathrm{g})$, fruit length $(\mathrm{cm})$, fruit diameter $(\mathrm{cm})$, number of seeds per fruit, fruit fly infestation $\%$, yield of marketable fruits per plant $(\mathrm{g})$, TSS ( ${ }^{0}$ Brix), ascorbic acid $(\mathrm{mg} / 100 \mathrm{~g})$, and chlorophyll content (mg/100gm) carotenoid content (mg/100gm) and total phenol content.

The present experiment was conducted to estimate the level of heterosis per cent over better parent and standard variety among crosses F1 hybrids of seven varieties. This knowledge would be useful to investigate the performance and relationship of F1 hybrids and parents and to select suitable parents and population for designing an effective crop 
improvement programme. The heterosis was determined as per cent increase or decrease of $\mathrm{F} 1$ values over the better parent (BP) and the standard variety. Data were taken for both consecutive year 2016 and 2017 and the pooled mean data was subjected to calculation of heterosis. These were calculated the method suggested by Falconer and Mackay (1996).

\section{Results and Discussion}

The negative magnitude of heterosis were considered desirable for node number of $1^{\text {st }}$ male flower appears, node number of $1^{\text {st }}$ female flower appears, days to $1^{\text {st }}$ male flower appears, days to $1^{\text {st }}$ female flower appears, days to $50 \%$ flowering, internodal length, fruit andfruit fly Infestation \%. However, for the rest of characters, positive estimates of heterosis were desirable.

Magnitude of heterosis over BP (better parent) and SV (standard variety) for different characters was estimated and given in Table No. 01

For node number of $1^{\text {st }}$ male flower appears, heterosis over better parent ranged varied from $-24.48 \%$ to $5.44 \%$ while, standard heterosis for this trait varied from -30.17 to $4.29 \%$. Out of 21 hybrids only 7 hybrids exhibited highly significant negative heterosis over better parent whereas, 10 hybrids was found to contain highly significant negative heterosis in desirable direction over standard check variety NBGH 167.

The hybrid Pusa Rasdar X Konkan Tara exhibited maximum heterosis over standard variety for this trait.For node number at which $1^{\text {st }}$ female flower five hybrids exhibited highly significant negative heterosis over better parent whereas, six hybrids was found to contain highly significant negative heterosis in desirable direction over standard check variety NBGH 167 . For days taken to $1^{\text {st }}$ female flowering two hybrids was found to contain highly significant negative heterosis over better parent, whereas, five hybrids was exhibited highly significant negative heterosis over standard variety in desirable direction.

For days taken to $50 \%$ flowering, only three hybrids was found to contain significant negative heterosis over better parent, whereas, only one hybrid namely Pusa Ausadhi X BRBT Local (-6.17 \%) showed negative significant heterosis in desirable direction over standard variety. Similar findings of negative and high heterosis for these traits were reported by Jadhav et al., (2009) Laxuman et al., (2012) and Bhatt et al., (2016).

For internodal length the extent of heterosis over better parent range varied from $-23.89 \%$ (PusaRasdar X BRBT Local) to $12.42 \%$ (Jhalri X BRBT Local). The economic heterosis for this trait varied from $-23.04 \%$ (PusaAusadhi X Konkan Tara) to $23.30 \%$ (Jhalri X BRBT Local). Six hybrids were exhibited highly significant negative heterosis over better parent whereas, nine hybrids showed highly significant negative heterosis over standard check in desirable direction. Short internodal length may cause maximum number of node number of male or female flower and may participate more number of fruits per plant and cause more yield.

For number of primary branch per plant only one hybrid i.e., PusaAusadhi X BRBT Local $(8.51 \%)$ was found to contain highly significant positive heterosis over better parent, and fourteen hybrids was exhibited highly significant positive heterosis over standard variety in desirable direction. Similar result was supported by Tewari and Ram (2001). Heterosis regarding growth parameters is related to earliness, high vigour and yield and thus they are strongly 
associated. The hybrid Kathi No.1 and BRBT Local $(39.25 \%)$ followed by PusaAusadhi (33.99\%) were showed maximum vine length among all the hybrids. This result was also reported by Singh et al., (2013) and Kadansamy (2015). The ideal plant type should have maximum vine length, maximum number of primary branches which may leads higher yields (Sirohi and Choudhury, 1978).

Yield attributing traits i.e., fruit length, fruit diameter, fruit weight, number of fruits per vine play pivotal role in yield of any crop. Hence these traits positive heterosis is desirable. For fruit length, out of 21 hybrids, four hybrids was found to contain significant positive heterosis over better parent, whereas, five hybrids exhibited highly significant positive heterosis over standard variety in desirable direction. The cross PusaAusadhi $\mathrm{X}$ BRBT Local showed maximum positive and significant heterosis $(29.34 \%)$ over standard check. Similar results were recorded by Ranpise et al., (1992) for standard heterosis (26.02\%) in bitter gourd.

For fruit weight only two hybrids, were exhibited highly significant positive heterosis over better parent whereas, six hybrids showed highly significant positive heterosis over standard check in desirable direction.

The cross PusaAusadhi $\times$ BRBT Local showed significant and positive heterosis of (31.92\%) over mid parent, 10.55 per cent over better parent and 44.23 per cent showed positive and significant heterosis of over the commercial check. Similar results were reported by Singh and Kumar (2002), Yadav et al., (2009) and Laxman et al., (2012).

For fruit diameter, the cross combination PusaRasdar X BRBT Local (38.94 \%) showed highly significant positive heterosis over standard variety. Similar finding were reported by Laxman et al., (2012) in the crosses PusaVishesh X IC 68310 (38.10\%) for fruit diameter. For number of fruits per plant, the cross combination PusaAusadhi X Konkan Tara showed maximum and positive heterosis $28.21 \%$ over better parent. Rainpise et al., observed 32.70 percent, $9.0 \%$ by Mishra et al., (1994) and $53.84 \%$ by Singh et al., (2001) for standard heterosis in bitter gourd.

The one of the important parameters like fruit yield per vine, our results exhibited highly significant positive heterosis in desirable direction, six hybrid over better parent and six hybrids over standard variety out of 21 hybrids which shown positive as well as high significant heterosis. The hybrid PusaAusadhi $X$ BRBT Local exhibited maximum heterosis over better parent $(25.38 \%)$ and $(26.88 \%)$ over standard check respectively. Our results are also conformity with the results of Mishra et al., 1999 Jadav et al., 2009 and Thangamani and Pugalendhi (2013).

The other traits like number of seeds per fruit which was varied from -29.62 per cent (Kathi No.1 X PusaRasdar) to $38.57 \%$ (BRBT Local X Konkan Tara). While as, Heterosis over better parent which was ranged from -42.98 $\%$ (Konkan Tara X Solan Hara) to $8.78 \%$ (BRBT Local X Konkan Tara). Similar results were found by earlier scientist Celine and Sirohi (1996).

Data regarding to Fruit fly infestation, our results confined that therelative heterosis ranged from $2.43 \%$ (Kathi No.1 X Solan Hara) to $31.56 \%$ (Kathi No.1 X Konkan Tara) respectively. While as, Heterobeltiosis ranged was noted from $-2.26 \%$ (PusaAusadhi $\mathrm{X}$ BRBT Local) to $25.19 \%$ (Kathi No.1 X BRBT Local) respectively.

The important traits regarding to biochemical attributes; such as TSS, Ascorbic acid, total chlorophyll content, Carotenoids and total phenolic compound. 
Table.1 Estimation of heterosis for quantitative and qualitative traits of bitter gourd

\begin{tabular}{|c|c|c|c|c|c|c|c|c|c|c|}
\hline \multirow{2}{*}{$\begin{array}{l}\text { Characters } \\
\text { Hybrids }\end{array}$} & \multicolumn{2}{|c|}{$\begin{array}{l}\text { Node Number at 1st male } \\
\text { flower appears }\end{array}$} & \multicolumn{2}{|c|}{$\begin{array}{l}\text { Node Number at 1st Female } \\
\text { flower appears }\end{array}$} & \multicolumn{2}{|c|}{$\begin{array}{c}\text { Days to Ist Male flower } \\
\text { appears }\end{array}$} & \multicolumn{2}{|c|}{$\begin{array}{l}\text { Days to Ist Female } \\
\text { flower appears }\end{array}$} & \multicolumn{2}{|c|}{$\begin{array}{l}\text { Days to 50\% } \\
\text { Flowering }\end{array}$} \\
\hline & BPH & SH & $\mathrm{BPH}$ & $\mathrm{SH}$ & BPH & SH & $\mathrm{BPH}$ & SH & $\mathrm{BPH}$ & $\mathrm{SH}$ \\
\hline PusaAusadhi X Jhalri & $-24.48 * *$ & $-25.98 * *$ & $-17.47 * *$ & $-14.28 * *$ & 1.51 & -4.60 & -6.92 & $-11.51 *$ & -1.88 & -3.71 \\
\hline PusaAusadhi X Kathi No. 01 & 0.00 & -7.20 & 4.07 & -1.89 & -4.02 & $-7.09 *$ & $-13.72 * *$ & $-15.14 * *$ & -3.99 & -3.40 \\
\hline PusaAusadhi X PusaRasdar & 6.08 & $-27.02 * *$ & 3.56 & $-17.14 * *$ & 4.97 & -2.84 & -5.97 & $-11.85^{* *}$ & $-7.75^{*}$ & -5.48 \\
\hline PusaAusadhi X BRBT Local & $-23.48 * *$ & $-21.82 * *$ & $-25.21 * *$ & $-15.22 * *$ & 0.00 & -3.89 & -4.07 & -6.91 & $-10.05 * *$ & $-6.17 *$ \\
\hline PusaAusadhi X Konkan Tara & $-12.23 * *$ & $-24.95 * *$ & -7.70 & $-19.99 * *$ & -3.96 & -5.32 & $-15.51 * *$ & $-15.79 * *$ & 1.24 & 0.29 \\
\hline PusaAusadhi X Solan Hara & $-17.39 * *$ & $-20.75 * *$ & $-25.65 * *$ & $-19.97 * *$ & 5.39 & -3.20 & -5.09 & $-14.15^{* *}$ & -1.80 & 0.31 \\
\hline Jhalri X Kathi No. 01 & 3.19 & 1.15 & 5.49 & $9.56^{*}$ & 1.84 & -1.42 & -1.44 & -3.06 & 1.22 & 1.84 \\
\hline Jhalri X PusaRasdar & $-9.58 *$ & $-11.37 * *$ & -5.53 & -1.89 & 0.38 & -5.66 & 0.69 & -4.28 & -3.61 & -1.24 \\
\hline Jhalri X BRBT Local & -5.10 & -3.05 & -0.83 & $12.41 * *$ & -1.85 & -5.67 & -1.36 & -4.28 & $-6.21 *$ & -2.16 \\
\hline Jhalri X Konkan Tara & $-8.52 *$ & $-10.33 * *$ & -1.86 & 1.92 & -1.80 & -3.19 & -4.28 & -4.61 & 1.86 & 0.91 \\
\hline Jhalri X Solan Hara & -2.12 & -4.06 & 0.90 & 8.61 & 4.53 & -1.76 & 2.42 & -2.63 & -3.31 & -1.24 \\
\hline Kathi No. 01 X PusaRasdar & 4.47 & -3.05 & 0.01 & -5.72 & -3.29 & $-6.38 *$ & -4.69 & -6.26 & -1.81 & 0.61 \\
\hline Kathi No. 01 X BRBT Local & 2.07 & 4.29 & -6.72 & 5.74 & 0.38 & -2.83 & -2.00 & -3.62 & -1.77 & 2.47 \\
\hline Kathi No. 01 X Konkan Tara & 2.26 & -5.10 & 6.07 & 0.00 & -0.72 & -2.12 & -1.32 & -1.65 & 2.76 & 3.39 \\
\hline Kathi No. 01 X Solan Hara & 5.44 & 1.15 & 2.66 & $10.50 *$ & 1.48 & -1.76 & -2.33 & -3.94 & 0.92 & 3.09 \\
\hline PusaRasdar X BRBT Local & $-20.39 * *$ & $-18.67 * *$ & $-17.66 * *$ & -6.66 & 4.43 & 0.37 & 2.71 & -0.34 & -3.84 & 0.31 \\
\hline PusaRasdar X Konkan Tara & $-18.34 * *$ & $-30.17 * *$ & -5.51 & $-18.08 * *$ & 3.24 & 1.78 & -1.98 & -2.31 & 0.00 & 2.47 \\
\hline PusaRasdar X Solan Hara & $-16.31 * *$ & $-19.71 * *$ & $-12.42 * *$ & -5.73 & 4.59 & -3.20 & 2.11 & -4.28 & -2.71 & -0.31 \\
\hline BRBT Local X Konkan Tara & -5.10 & -3.05 & -3.35 & $9.56^{*}$ & 2.16 & 0.72 & 0.66 & 0.33 & -2.07 & 2.16 \\
\hline BRBT Local X Solan Hara & -4.06 & -1.98 & 3.35 & $17.15^{* *}$ & -1.11 & -4.95 & -3.40 & -6.26 & 1.47 & 5.85 \\
\hline Konkan Tara X Solan Hara & -5.47 & $-9.31 *$ & 1.78 & $9.56^{*}$ & 0.00 & -1.41 & -2.64 & -2.96 & -3.01 & -0.93 \\
\hline Comparison of Hybrid with & C.D. $95 \%$ & C.D. $99 \%$ & C.D. $95 \%$ & C.D. 99\% & C.D. $95 \%$ & C.D. $99 \%$ & C.D. $95 \%$ & C.D. $99 \%$ & $\begin{array}{l}\text { C.D. } \\
\text { 95\% }\end{array}$ & $\begin{array}{l}\text { C.D. } \\
\text { 99\% }\end{array}$ \\
\hline Better Parent & & 0.608 & 0.811 & 2.127 & 1.388 & 3.639 & 2.260 & 5.926 & 1.659 & 4.349 \\
\hline Checks & & 0.608 & 0.811 & 2.127 & 1.388 & 3.639 & 2.260 & 5.926 & 1.659 & 4.349 \\
\hline
\end{tabular}




\begin{tabular}{|c|c|c|c|c|c|c|c|c|c|c|}
\hline \multirow{3}{*}{$\begin{array}{l}\text { Cont... } \\
\text { Characters } \\
\text { Hybrids }\end{array}$} & \multirow{2}{*}{\multicolumn{2}{|c|}{ Inter Nodal Length (cm) }} & \multirow{2}{*}{\multicolumn{2}{|c|}{$\begin{array}{c}\text { Primary Branches per } \\
\text { plant }\end{array}$}} & \multirow{2}{*}{\multicolumn{2}{|c|}{$\begin{array}{c}\text { Number of fruits per } \\
\text { plant }\end{array}$}} & \multirow{2}{*}{\multicolumn{2}{|c|}{ Fruit Length (cm) }} & \multirow{2}{*}{\multicolumn{2}{|c|}{ Fruit Weight }} \\
\hline & & & & & & & & & & \\
\hline & BPH & SH & BPH & SH & BPH & SH & $\mathrm{BPH}$ & $\mathrm{SH}$ & $\mathrm{BPH}$ & $\mathrm{SH}$ \\
\hline PusaAusadhi X Jhalri & $-15.16^{* *}$ & -6.95 & $-10.18^{*}$ & $22.38 * *$ & -3.88 & $18.64 * *$ & 0.21 & 2.51 & 4.33 & -7.97 \\
\hline $\begin{array}{l}\text { PusaAusadhi X Kathi No. } \\
\text { 01 }\end{array}$ & -4.82 & $-9.45^{*}$ & -7.63 & $25.85 * *$ & 3.43 & $27.66 * *$ & 2.64 & -2.51 & 9.64 & -3.29 \\
\hline PusaAusadhi X PusaRasdar & 4.61 & $-12.87 * *$ & $-15.21 * *$ & $15.52 * *$ & -6.90 & $14.91 * *$ & $-12.51 *$ & $-16.91 * *$ & -2.34 & 0.29 \\
\hline PusaAusadhi X BRBT Local & $-20.15^{* *}$ & $-14.11 * *$ & $8.51 *$ & $53.44 * *$ & $-32.78 * *$ & $-17.03 * *$ & $9.27 *$ & $29.24 * *$ & $10.55 * *$ & $44.23 * *$ \\
\hline $\begin{array}{l}\text { PusaAusadhi X Konkan } \\
\text { Tara }\end{array}$ & -4.47 & $-23.04 * *$ & $-11.43 * *$ & $20.68 * *$ & -5.50 & $28.21 * *$ & -5.41 & $-10.17 *$ & -0.58 & $-12.31 *$ \\
\hline PusaAusadhi X Solan Hara & $-14.83 * *$ & $-20.13 * *$ & $-16.50 * *$ & $13.76^{*}$ & -6.47 & $15.44 * *$ & $-15.66^{* *}$ & $-19.90 * *$ & -2.61 & $-14.10 * *$ \\
\hline Jhalri X Kathi No. 01 & 2.91 & $12.87 * *$ & -5.48 & $18.92 * *$ & 0.43 & $23.95 * *$ & 6.30 & 8.74 & -8.70 & $-19.78 * *$ \\
\hline Jhalri X PusaRasdar & $-18.94 * *$ & $-11.10^{*}$ & $-12.10 *$ & 0.00 & $-15.53 * *$ & 4.26 & $10.11^{*}$ & $12.64 * *$ & $-10.50^{*}$ & -8.08 \\
\hline Jhalri X BRBT Local & $12.42 * *$ & $23.30 * *$ & $-18.30 * *$ & $15.52 * *$ & -0.86 & $22.35 * *$ & -1.71 & $16.26^{* *}$ & $\begin{array}{c}- \\
36.52 * *\end{array}$ & $-17.18 * *$ \\
\hline Jhalri X Konkan Tara & -2.79 & 6.62 & -7.83 & 1.71 & $-8.63 *$ & $23.96 * *$ & $-10.17 *$ & -8.11 & 6.81 & $-22.27 * *$ \\
\hline Jhalri X Solan Hara & -5.21 & 3.96 & 1.70 & 5.17 & -1.29 & $21.83 * *$ & 0.46 & 2.76 & -3.36 & $-29.14 * *$ \\
\hline Kathi No. 01 X PusaRasdar & $-9.96^{*}$ & $-14.34 * *$ & 5.52 & $32.76^{* *}$ & 7.34 & $24.47 * *$ & $23.60 * *$ & -4.79 & $\begin{array}{c}- \\
13.96^{* *}\end{array}$ & $-11.64 *$ \\
\hline Kathi No. 01 X BRBT Local & -4.07 & 3.19 & 7.30 & $51.73 * *$ & -3.20 & $12.25^{*}$ & -4.69 & $12.73 * *$ & 0.51 & $31.13 * *$ \\
\hline $\begin{array}{l}\text { Kathi No. } 01 \text { X Konkan } \\
\text { Tara }\end{array}$ & -0.57 & -5.41 & 1.38 & $27.56^{* *}$ & $-18.84 * *$ & 10.11 & 10.44 & $-14.94 * *$ & 9.99 & -3.36 \\
\hline Kathi No. 01 X Solan Hara & 4.33 & -0.75 & -8.18 & $15.52 * *$ & -5.05 & 10.11 & $13.37 *$ & $-12.68 * *$ & 2.47 & -9.97 \\
\hline PusaRasdar X BRBT Local & $-23.89 * *$ & $-18.13 * *$ & $-12.24 * *$ & $24.09 * *$ & -5.27 & $-13.83 * *$ & $-14.39 * *$ & 1.26 & -1.93 & $27.96 * *$ \\
\hline PusaRasdar X Konkan Tara & 3.68 & $-13.65 * *$ & -3.05 & 10.29 & $-35.70 * *$ & $-12.76^{*}$ & -2.62 & $-26.75 * *$ & $15.34 * *$ & $18.45 * *$ \\
\hline PusaRasdar X Solan Hara & $11.45^{*}$ & 4.51 & -3.06 & 10.28 & -5.71 & -3.71 & 3.56 & $-22.10 * *$ & -0.92 & 1.75 \\
\hline $\begin{array}{l}\text { BRBT Local X Konkan } \\
\text { Tara }\end{array}$ & 3.04 & $10.84 *$ & 1.21 & $43.11 * *$ & $-21.98 * *$ & 5.85 & 5.46 & $24.73 * *$ & -5.35 & $23.49 * *$ \\
\hline BRBT Local X Solan Hara & $10.03^{*}$ & $18.36 * *$ & $-10.98 * *$ & $25.88 * *$ & $-23.43 * *$ & $-21.81 * *$ & -4.94 & $12.44^{*}$ & $-9.65^{*}$ & $17.88 * *$ \\
\hline Konkan Tara X Solan Hara & $-12.85^{* *}$ & $-18.28 * *$ & 1.56 & $12.07 *$ & $-14.52 * *$ & $15.96 * *$ & 2.01 & $-24.96 * *$ & 13.31 & -16.93 \\
\hline Comparison of Hybrid with & C.D. $95 \%$ & C.D. 99\% & C.D. $95 \%$ & C.D. $99 \%$ & C.D. $95 \%$ & C.D. $99 \%$ & $\begin{array}{l}\text { C.D. } \\
\text { 95\% }\end{array}$ & $\begin{array}{l}\text { C.D. } \\
\text { 99\% }\end{array}$ & $\begin{array}{l}\text { C.D. } \\
\text { 95\% }\end{array}$ & $\begin{array}{l}\text { C.D. } \\
\text { 99\% }\end{array}$ \\
\hline Better Parent & 0.291 & 0.762 & 0.563 & 1.477 & 1.644 & 4.311 & 0.664 & 1.742 & 3.194 & 8.375 \\
\hline Checks & 0.291 & 0.762 & 0.563 & 1.477 & 1.644 & 4.311 & 0.664 & 1.742 & 3.194 & 8.375 \\
\hline
\end{tabular}




\begin{tabular}{|c|c|c|c|c|c|c|c|c|c|c|}
\hline \multicolumn{11}{|l|}{ Cont... } \\
\hline Characters & \multicolumn{2}{|c|}{ Fruit Diameter } & \multicolumn{2}{|c|}{ Number of seed per fruit } & \multicolumn{2}{|c|}{ Vine Length } & \multicolumn{2}{|c|}{ Yield/Vine } & \multicolumn{2}{|c|}{ Fruit Fly Infestation } \\
\hline Hybrids & $\mathrm{BPH}$ & SH & BPH & SH & $\mathrm{BPH}$ & SH & $\mathrm{BPH}$ & SH & $\mathrm{BPH}$ & SH \\
\hline PusaAusadhi X Jhalri & 2.41 & -0.95 & $-19.37 * *$ & $-17.62 * *$ & -7.66 & 8.02 & 6.29 & 7.56 & -1.53 & -7.19 \\
\hline PusaAusadhi X Kathi No. 01 & $-15.35^{* *}$ & 2.00 & $-35.83 * *$ & $-15.39 * *$ & $-17.03 * *$ & 1.70 & $14.93 * *$ & $16.30 * *$ & $15.03 * *$ & -8.04 \\
\hline PusaAusadhi X PusaRasdar & $-13.57 * *$ & $20.54 * *$ & $-23.28 * *$ & $-27.51 * *$ & 0.17 & -3.47 & $11.70 * *$ & $13.03 * *$ & 3.44 & $-17.31 * *$ \\
\hline PusaAusadhi X BRBT Local & 2.44 & $37.95 * *$ & $-12.28 * *$ & $9.88^{*}$ & 3.93 & $33.99 * *$ & $25.38 * *$ & $26.88 * *$ & -2.26 & $-21.87 * *$ \\
\hline PusaAusadhi X Konkan Tara & -1.57 & -4.37 & $-20.95 * *$ & $-25.30 * *$ & -7.27 & -6.31 & $14.33 * *$ & $15.69 * *$ & 0.87 & $-19.36 * *$ \\
\hline PusaAusadhi X Solan Hara & -6.00 & -9.08 & $-24.21 * *$ & 6.59 & -3.56 & $-12.23 *$ & -6.77 & -5.65 & $24.14 * *$ & -0.76 \\
\hline Jhalri X Kathi No. 01 & $-22.73 * *$ & -6.89 & $-9.14^{*}$ & $19.79 * *$ & -4.35 & $17.24 * *$ & 0.91 & -1.89 & 1.42 & -4.41 \\
\hline Jhalri X PusaRasdar & $-21.58 * *$ & 9.37 & $-30.11 * *$ & $-28.59 * *$ & 4.28 & $21.99 * *$ & 1.64 & $-9.32 *$ & 2.36 & -3.52 \\
\hline Jhalri X BRBT Local & $-26.55 * *$ & -1.09 & $-21.93 * *$ & -2.21 & -2.55 & $25.64 * *$ & -1.91 & -1.24 & 8.58 & 2.34 \\
\hline Jhalri X Konkan Tara & -5.34 & -8.04 & $-34.40 * *$ & $-32.97 * *$ & -4.13 & $12.16^{*}$ & 0.80 & -4.30 & -1.19 & -6.87 \\
\hline Jhalri X Solan Hara & -7.33 & $-12.89 * *$ & $-30.47 * *$ & -2.21 & 1.70 & $18.97 * *$ & 0.44 & $-16.86^{* *}$ & 8.99 & 2.73 \\
\hline Kathi No. 01 X PusaRasdar & $-24.04 * *$ & 5.94 & $-42.52 * *$ & $-24.21 * *$ & $-14.19 * *$ & 5.18 & $10.32 *$ & 7.26 & $25.09 * *$ & $-13.10 * *$ \\
\hline Kathi No. 01 X BRBT Local & 0.88 & $35.85 * *$ & $-14.16 * *$ & $13.18 * *$ & 8.02 & $39.25 * *$ & $23.99 * *$ & 24.83 & $25.19 * *$ & $-13.03 * *$ \\
\hline Kathi No. 01 X Konkan Tara & $-22.89 * *$ & -7.09 & $-30.00 * *$ & -7.70 & $-16.27 * *$ & 2.64 & 7.94 & 4.95 & $24.00 * *$ & $-13.86 * *$ \\
\hline Kathi No. 01 X Solan Hara & $-15.79 * *$ & 1.47 & $-10.15 * *$ & $26.38 * *$ & $-10.17 *$ & 10.11 & -2.65 & -5.35 & -2.07 & $-25.41 * *$ \\
\hline PusaRasdar X BRBT Local & -0.38 & $38.94 * *$ & $-29.82 * *$ & $-12.09 *$ & $-17.48 * *$ & 6.39 & 1.11 & 1.81 & $23.48 * *$ & $-17.84 * *$ \\
\hline PusaRasdar X Konkan Tara & $-13.19 * *$ & $21.07 * *$ & $-22.39 * *$ & $-35.18 * *$ & 2.42 & 3.48 & 2.00 & -3.15 & 9.43 & $-27.19 * *$ \\
\hline PusaRasdar X Solan Hara & $-13.81 * *$ & $20.21 * *$ & $-42.21 * *$ & $-18.72 * *$ & -7.86 & -11.21 & -1.13 & $-11.79 * *$ & $11.57 *$ & $-15.01 * *$ \\
\hline BRBT Local X Konkan Tara & -5.97 & $26.63 * *$ & $8.78 *$ & $36.27 * *$ & 1.21 & $30.49 * *$ & $22.30 * *$ & $23.14 * *$ & 8.01 & $-30.79 * *$ \\
\hline BRBT Local X Solan Hara & -3.32 & $30.19 * *$ & $7.04 *$ & $50.56^{* *}$ & -0.38 & $28.43 * *$ & -2.93 & -2.27 & $15.35 * *$ & $-12.14 * *$ \\
\hline Konkan Tara X Solan Hara & -9.20 & $-11.79 *$ & $-42.98 * *$ & $-19.79 * *$ & 6.33 & 7.43 & -0.80 & -5.82 & $19.31 * *$ & $-9.12 *$ \\
\hline Comparison of Hybrid with & C.D. $95 \%$ & C.D. $99 \%$ & C.D. $95 \%$ & C.D. $99 \%$ & C.D. $95 \%$ & C.D. $99 \%$ & $\begin{array}{l}\text { C.D. } \\
95 \%\end{array}$ & $\begin{array}{l}\text { C.D. } \\
\text { 99\% }\end{array}$ & $\begin{array}{l}\text { C.D. } \\
95 \%\end{array}$ & $\begin{array}{l}\text { C.D. } \\
99 \%\end{array}$ \\
\hline Better Parent & 0.148 & 0.387 & 0.620 & 1.625 & 10.695 & 28.043 & 7.298 & 19.137 & 1.000 & 2.622 \\
\hline Checks & 0.171 & 0.447 & 0.716 & 1.877 & 12.349 & 32.381 & 8.427 & 22.097 & 1.155 & 3.028 \\
\hline
\end{tabular}


Int.J.Curr.Microbiol.App.Sci (2020) 9(2): 1614-1623

\begin{tabular}{|c|c|c|c|c|c|c|c|c|c|c|}
\hline \multirow{2}{*}{$\begin{array}{l}\text { Characters } \\
\text { Hybrids }\end{array}$} & \multicolumn{2}{|c|}{ Total Soluble Solids (TSS) } & \multicolumn{2}{|c|}{ Ascorbic Acid } & \multicolumn{2}{|c|}{ Total chlorophyll } & \multicolumn{2}{|c|}{ Carotenoids } & \multicolumn{2}{|c|}{ Total Phenol } \\
\hline & $\mathrm{BPH}$ & $\mathrm{SH}$ & $\mathrm{BPH}$ & SH & BPH & $\mathrm{SH}$ & $\mathrm{BPH}$ & $\mathrm{SH}$ & $\mathrm{BPH}$ & $\mathrm{SH}$ \\
\hline PusaAusadhi X Jhalri & $-9.02 * *$ & $13.35^{* *}$ & -6.81 & $-20.47 * *$ & $-23.14 * *$ & $-23.14 * *$ & 0.86 & $59.14 * *$ & 4.85 & $-6.16^{* *}$ \\
\hline $\begin{array}{l}\text { PusaAusadhi X Kathi No. } \\
01\end{array}$ & -1.15 & $8.16^{*}$ & $-18.82 * *$ & $-7.81 * *$ & $-24.37 * *$ & $-24.37 * *$ & $-6.85 * *$ & $71.98 * *$ & $-7.21 * *$ & 0.29 \\
\hline PusaAusadhi X PusaRasdar & $-7.29 *$ & 1.45 & $-17.46^{* *}$ & $-17.08 * *$ & 2.34 & 2.34 & -4.81 & $50.19 * *$ & -2.37 & -2.73 \\
\hline PusaAusadhi X BRBT Local & $-7.73 *$ & $24.81 * *$ & $-34.09 * *$ & $-11.98 * *$ & $-21.58 * *$ & $-21.58 * *$ & $-24.29 * *$ & $19.46^{* *}$ & 1.53 & $-9.14 * *$ \\
\hline $\begin{array}{l}\text { PusaAusadhi X Konkan } \\
\text { Tara }\end{array}$ & -2.04 & $10.60 * *$ & $-27.30 * *$ & 0.24 & $-29.51 * *$ & $-29.51 * *$ & -1.16 & $98.25^{* *}$ & -4.31 & -0.41 \\
\hline PusaAusadhi X Solan Hara & 2.10 & $11.72 * *$ & $-10.90 * *$ & $-15.61 * *$ & $-11.05^{*}$ & $-11.05^{*}$ & $-11.10 * *$ & $40.27 * *$ & -3.04 & $-13.22 * *$ \\
\hline Jhalri X Kathi No. 01 & 5.27 & $31.15^{* *}$ & $-14.50 * *$ & -2.90 & $-28.83 * *$ & $-28.83 * *$ & $-40.25 * *$ & $10.31 *$ & $18.99^{* * *}$ & $-12.44 * *$ \\
\hline Jhalri X PusaRasdar & 3.39 & $28.81 * *$ & -5.91 & -5.48 & 3.61 & 3.61 & $20.75 * *$ & $36.96 * *$ & $\begin{array}{c}- \\
20.12 * *\end{array}$ & $-20.41 * *$ \\
\hline Jhalri X BRBT Local & $12.94 * *$ & $52.76 * *$ & $-27.86 * *$ & -3.67 & $-8.71 * *$ & $-8.71 * *$ & 5.25 & -6.42 & -1.83 & $-20.90 * *$ \\
\hline Jhalri X Konkan Tara & 2.14 & $27.25 * *$ & $-27.53 * *$ & -0.08 & $-21.87 * *$ & $-21.87 * *$ & $-33.27 * *$ & $33.85 * *$ & $\overline{-}^{-}$ & $-9.05 * *$ \\
\hline Jhalri X Solan Hara & -2.02 & $22.06 * *$ & -6.43 & $-11.39 * *$ & $-11.68 * *$ & $-11.68 * *$ & 3.29 & $9.92 *$ & -0.93 & $-28.05 * *$ \\
\hline Kathi No. 01 X PusaRasdar & 2.15 & $10.83 * *$ & -3.58 & $9.51 * *$ & $-18.87 * *$ & $-18.87 * *$ & -0.84 & $83.07 * *$ & 2.53 & $10.82 * *$ \\
\hline Kathi No. 01 X BRBT Local & -4.11 & $29.70 * *$ & 1.89 & $36.05 * *$ & 2.07 & 2.07 & $-7.48 * *$ & $70.82 * *$ & $28.41 * *$ & $-22.62 * *$ \\
\hline $\begin{array}{l}\text { Kathi No. } 01 \text { X Konkan } \\
\text { Tara }\end{array}$ & -2.59 & $9.97 *$ & 4.46 & $44.02 * *$ & $-9.36 * *$ & $-9.36 * *$ & $29.10 * *$ & $158.95 * *$ & $7.21 * *$ & $15.87 * *$ \\
\hline Kathi No. 01 X Solan Hara & 2.87 & $11.61 * *$ & 0.37 & $13.99 * *$ & $-41.31 * *$ & $-41.31 * *$ & $10.33 * *$ & $103.70 * *$ & $-5.99 * *$ & 1.60 \\
\hline PusaRasdar X BRBT Local & $-20.83 * *$ & 7.08 & $-7.74 * *$ & $23.20 * *$ & $-16.87 * *$ & $-16.87 * *$ & $20.58 * *$ & $36.77 * *$ & -1.90 & -2.26 \\
\hline PusaRasdar X Konkan Tara & 4.63 & $18.13 * *$ & $-8.48 * *$ & $26.18 * *$ & $-25.99 * *$ & $-25.99 * *$ & 3.30 & $107.20 * *$ & 0.14 & 4.22 \\
\hline PusaRasdar X Solan Hara & -5.97 & -1.93 & $22.81 * *$ & $23.37 * *$ & $-7.24 *$ & $-7.24 *$ & $31.90 * *$ & $49.61 * *$ & 2.54 & 2.16 \\
\hline $\begin{array}{l}\text { BRBT Local X Konkan } \\
\text { Tara }\end{array}$ & 1.64 & $37.49 * *$ & $15.10 * *$ & $58.68 * *$ & $9.68 * *$ & $9.68 * *$ & $-12.42 * *$ & $75.68 * *$ & -3.68 & 0.23 \\
\hline BRBT Local X Solan Hara & $6.47 *$ & $44.01 * *$ & 1.67 & $35.76 * *$ & $-12.59 * *$ & $-12.59 * *$ & $21.39 * *$ & $29.18 * *$ & -0.57 & $-19.88 * *$ \\
\hline Konkan Tara X Solan Hara & $10.05 * *$ & $24.25^{* *}$ & $8.50 * *$ & $49.59 * *$ & $-16.36 * *$ & $-16.36 * *$ & $15.13^{* *}$ & $130.93 * *$ & $5.38^{*}$ & $9.67 * *$ \\
\hline Comparison of Hybrid with & C.D. $95 \%$ & C.D. 99\% & C.D. $95 \%$ & C.D. $99 \%$ & C.D. $95 \%$ & C.D. $99 \%$ & $\begin{array}{l}\text { C.D. } \\
95 \%\end{array}$ & $\begin{array}{l}\text { C.D. } \\
\text { 99\% }\end{array}$ & $\begin{array}{l}\text { C.D. } \\
95 \%\end{array}$ & $\begin{array}{l}\text { C.D. } \\
99 \%\end{array}$ \\
\hline Better Parent & 0.155 & 0.407 & 1.873 & 4.911 & 0.022 & 0.057 & 0.032 & 0.084 & 2.534 & 6.646 \\
\hline Checks & 0.179 & 0.470 & 2.163 & 5.671 & 0.025 & 0.066 & 0.037 & 0.097 & 2.927 & 7.674 \\
\hline
\end{tabular}


In better parents heterosis of bitter gourd, the range were varied from $-20.83 \%$ (PusaRasdar X BRBT Local) to $12.94 \%$ (Jhalri X BRBT Local) for TSS, Ascorbic acid ranged varied from $-34.09 \%$ (PusaAusadhi X BRBT Local) to $22.81 \%$ (PusaRasdar X Solan Hara), the total chlorophyll content varied from -41.31 $\%$ (Kathi No.1 X Solan Hara) to $9.68 \%$ (BRBT Local X Konkan Tara), the Carotenoids ranged varied from $-40.25 \%$ (Jhalri X Kathi No.1) to $31.90 \%$ (PusaRasdar $\mathrm{X}$ Solan Hara) and the total phenol contents ranged varied from -28.41\% (Kathi No.1 X BRBT Local) to $7.21 \%$ (Kathi No. $1 \mathrm{X}$ Konkan Tara) respectively.

In economic parents of heterosis of bitter gourd, the ranged varied from $-1.93 \%$ (PusaRasdar X Solan Hara) to $52.76 \%$ (Jhalri $\mathrm{X}$ BRBT Local) for TSS traits, Ascorbic acid varied from $-20.47 \%$ (PusaAusadhi X Jhalri) to $58.68 \%$ (BRBT Local X Konkan Tara), chlorophyll content varied from $-32.33 \%$ (PusaAusadhi X Solan Hara) to $70.91 \%$ (BRBT Local X Konkan Tara), carotenoid content varied from $-6.42 \%$ (Jhalri X BRBT Local) to $158.95 \%$ (Kathi No.1X Solan Hara) and the total phenolic contents varied from $28.05 \%$ (Jhalri X Solan Hara) to $15.87 \%$ (Kathi No.1 X Konkan Tara) respectively.

In findings related to biochemical attributes the similar results was recorded with earlier scientists Mamun et al., (2015) for Bcarotenoid, total soluble solid and phenol content, Behera et al., (2010) and Dhillon et al., (2016) for ascorbic acid, B-carotene in bitter gourd.

In conclusion the for crop improvement programme, heterosis breeding is one of the important tools to produce superior hybrids having qualities with maximum yield. From the present investigation it is concluded that the cross combination PusaAusadhi X BRBT Local exhibited maximum heterosis over standard check for yield and yield attributing trait like number of primary branch, vine length fruit length and fruit weight. In case of quality parameter, BRBT Local X Konkan Tara exhibit highest magnitude of heterosis for ascorbic acid and chlorophyll content.

\section{Acknowledgement}

The authors acknowledge, Chairman, Department of Horticulture, (Vegetables and Floriculture), Bihar Agricultural College, Bihar Agricultural University, Sabour, India for providing all the required infrastructure and facilities for the present work and those scientists whose published works has been cited in the text of this research paper.

\section{References}

Behera T K, Behera S and Bharath L K (2010). Bitter Gourd: Botany, Horticulture, Breeding Horticultural Reviews, Volume 37 Edited by Jules Janick. WileyBlackwell. Pp. 102-136.

BeheraTK (2004). Heterosis in bitter gourd. In: P K Singh, S K Dasgupta, and S K Thpathi (eds.), Hybrid vegetable development Haworth Press, New York, 217-221.

Bhatt L, Singh SP, Soni AK and Samota MK (2017) Combining Ability Studies in Bitter Gourd (Momordica charantia L.) for Quantitative Characters. International Journal of Current Microbiology Applied Science, 6(7): 4471-4478.

CelineVA and Sirohi PS (1996) Heterosis in bitter gourd (Momordica charantia L.). Veg. Sci., 28: 180-185

Dhillon, M.K., R. Singh, J.S. Naresh, and N.K. Sharma. 2005. Influence of physicochemicaltraits of bitter gourd, (Mom ordicachamntia L.)on lanai density and resistance to melon -fruit fly, Boctrocem cucurbitae (Coquillett). J. Appl. Entomol, 129:393-399

Falconer D S and Mackay T F C (1996). Introduction to Quantitative Genetics, 4th ed. Long man Scientific and Technical, London

Hayes, H. K., Immer, F. F. and Smith, D. C. (1956).Methods of plant breeding. 
McGraw Hill Book Publishing Company, Inc., New Delhi, India

Islam, MR, Hossain, MS, Bhuiyan, MSR, Husna, A and Syed, MA (2009) Genetic variability and path coefficient analysis of bitter gourd (Momordicacharantia). Internat. J. Sust. Agric.,1(3): 53-57

Jadhav KA, Garad BV, Dhumal, S. S., Kshirsagar, D. B., Patil, B. T. and Shinde K. G. (2009)Heterosis in bitter gourd. (Momordica charantia L.)Agri. Sci. Digest., 29: 7-11.

Kandasamy, R. (2015). Heterosis in bitter gourd (Momordica charantia L.). Asian J. Hort., 10(1): 158-160.

LaxumanPatil SA, Salimath PM, Dharmatti PR, Byadgi AS, Nirmalayenagi (2012) Heterosis and combining ability analysis for productivity traits in bitter gourd (Momordica charantia L.). Karnataka Journal Agriculture Science, 25: 9-13.

LaxumanPatil SA, Salimath PM,Dharmatti, PR (2012) Study on genetic diversity and its relation to heteropsis in bitter gourd (Momordica charantia L.). Karnataka J. Agric. Sci., 25 (1):14-17.

Mamuna, MHA, Rashid MH, Uddina N, Islama MR, and Asaduzzaman M (2015) Heterosis Studies in Bitter Gourd. International Journal of Vegetable Science, 22:5, 442-450

Miniraj, N., K.P. Prasanna, and K.V. Peter, 1993.Bitter gourd Mornordicuapp. pp. 239-246. In: C. Kalloo and 8.0. Bergh (ads.), Genetic improvement of vegetable plants. Pergamon Press, Oxford, UK.

Mishra HN, Mishra RS, Mishra SN and Parhi G (1994) Heterosis and combining ability in bitter gourd. Indian J. Agril. Sci., 64(5): 310-313.
Pal HP and Singh H. (1946). Studies in hybrid vigour. II. Notes on the manifestation of hybrid vigour in the brinjal and bitter gourd. Indian J. Genet. Plant Breed. 6:1933.

Rani R, Reddy R and Raju S C (2014) Inheritance of yield and its related traits in bitter gourd (MomordicacharantiaL.)Molecular Plant Breeding,5: 1-4.

Ranpise SA, Kale PN, Desale GY, Desai UT (1992) Heterosis in bitter gourd (Momordica charantia L.). South Indian Horticulture, 40: 313-15

Singh AK, Pan RS, Bhavana P (2013) Heterosis and combining ability analysis in bitter gourd (Momordica charantia L.). Supply Genetics Plant Breeding,8: 1533-36.

Singh DK, and Kumar Rajesh (2002) heterosis in bittergourd (Momordica charantia L.), progressive horticulture 34 (2): 204-207

Sirohi PS and Choudhury B. (1977) Combining ability in bitter gourd (Momordica charantia L.). Veg. Sci., 4(2): 107-115.

Tewari D, Ram HH and Jaiswal HR (2001) Studies on heterosis and combining ability in indigenous bitter gourd (Momordica charantia L.) for fruit yield. Vegetable Sciences, 28: 106-8.

Thangamani, C. and Pugalendhi, L. (2013a). Development of heterotic hybrids in bitter gourd (Momordica charantia L.) for earliness, high yield and quality traits. Asian Journal of Horticulture, 8: 195-201.

Turner, R.L. 1966. A comparative dictionary of the Indo-Aryan languages. Oxford University Press, London.

Walters TW, and DS Decker-Walters (1988). Balsam pear (Mormordica chorantia, Cucurbitaceae). Econ. Bot. 42: 286-286.

\section{How to cite this article:}

Amrita Kumari, Sangeeta Shree, Randhir Kumar, Chandan Kishore, V. K. Singh and Haque, M. 2020. Estimation of Heterosis for Yield and Quality traits in Bitter Gourd (Momordica charantia L.). Int.J.Curr.Microbiol.App.Sci. 9(02): 1614-1623.

doi: https://doi.org/10.20546/ijcmas.2020.902.185 